\title{
ACE2- Ang 1-7-MAS Axis in Human Diabetic Nephropathy
}

Sonoo Mizuiri' ${ }^{1 *}$, Yoshiko Nishizawa ${ }^{1}$, Makoto Hamanoue ${ }^{2}$, Hiromichi Hemmi ${ }^{3}$, Michitsune Arita ${ }^{3}$, Kazutoshi Shibuya ${ }^{4}$, Toshiyuki Aoki $^{5}$, Yasushi Ohashi ${ }^{5}$, Ken Sakai ${ }^{5}$ and Atsushi Aikawa ${ }^{5}$

${ }^{1}$ Division of Nephrology, Ichiyokai Harada Hospital, Hiroshima Japan

${ }^{2}$ Departments of Physiology, Toho University School of Medicine, Tokyo, Japan

${ }^{3}$ Departments of Molecular Biology, Toho University School of Medicine, Tokyo, Japan

${ }^{4}$ Departments of Surgical Pathology, Toho University School of Medicine, Tokyo, Japan

${ }^{5}$ Departments of Nephrology, Toho University School of Medicine, Tokyo, Japan

\begin{abstract}
The angiotensin-converting enzyme 2 (ACE2)- angiotensin-(1-7) [Ang (1-7)]-MAS receptor (MAS) axis might act as a counter-regulatory system against the angiotensin-converting enzyme (ACE)- angiotensin II (Ang II)-AT1 receptor (AT1) axis. We studied renal ACE/ACE2-Ang 1-7-MAS expression in human diabetic nephropathy. The subjects were 17 diabetic nephropathy (DN) patients, 17 healthy kidney donors, and 11 minimal change nephrotic syndrome (MCNS) patients as disease controls. Double immunofluorescent staining of kidney sections for ACE and ACE2, Ang 1-7 and ACE2, or MAS and ACE2 was performed, and the results were observed by confocal microscopy. For MAS, immunostaining, in situ hybridization, and RT-PCR were also performed. The percentage area that was positively immunostained for MAS and the intensity of the staining were evaluated by computerized imaging analysis. The median serum creatinine values of the DN patients, MCNS patients, and controls were $1.1 \mathrm{mg} / \mathrm{dl}, 0.8 \mathrm{mg} / \mathrm{dl}$, and $0.8 \mathrm{mg} / \mathrm{dl}$, respectively, and the median proteinuria values of the DN and MCNS patients were $3.7 \mathrm{~g} / \mathrm{day}$ and 6.8 $\mathrm{g} /$ day, respectively. ACE2 was mainly detected in the proximal tubules, but was also found in the glomeruli in all subjects. ACE2 co-localized with ACE, Ang 1-7, and MAS in the proximal tubules. Compared with the other specimens, the diabetic patients' proximal tubules displayed increased ACE and decreased ACE2 expression. Tubular Ang 1-7expression was downregulated in the DN patients compared with the controls and MCNS patients. Tubular MAS expression (density/pixel) was significantly $(\mathrm{P}<0.001)$ downregulated in the DN patients [7.33 $(6.26-9.68)]$ compared with the controls [24.51 (18.06-34.56)] and MCNS patients [23.75 (20.11-25.52)].
\end{abstract}

Conclusions: The tubular ACE2-Ang 1-7-MAS axis is downregulated in human diabetic nephropathy patients compared with healthy controls and MCNS patients.

Keywords: ACE2; Ang 1-7; MAS receptor; Human; Diabetic nephropathy

\section{Introduction}

Angiotensin-converting enzyme 2 (ACE2) was discovered in 2000. ACE2 is a homologue of angiotensin-converting enzyme (ACE) that forms angiotensin 1-7 (Ang 1-7) from Ang II and angiotensin 1-9 (Ang 1-9) from angiotensin I (Ang I) [1,2]. With regard to Ang 1-7, the identification of ACE2 and the demonstration that the MAS receptor (MAS) is involved in its actions have contributed to this heptapeptide being classified as a biologically active member of the RAS cascade [3]. The MAS is a receptor for Ang 1-7 [3]. Within the kidneys, Ang 1-7 is generated by ACE2-mediated degradation of Ang II; sequential cleavage of its precursor Ang I by ACE2 and ACE; or the actions of neprilysin, thimet oligopeptidase, prolyl oligopeptidase, or other peptidases against Ang I [4]. The recently identification of ACE2, Ang 1-7, and the MAS has led to conceptual changes in our understanding of the reninangiotensin system (RAS). There is a proposal that Ang 1-7 counteracts the vasoconstrictor, growth-promoting, and profibrotic action of Ang II [5]. Actually, ACE2, Ang 1-7, and MAS are expressed at high levels in the kidneys and have been reported to display renoprotective effects in animal studies [4-9].

However, the role of the ACE2-Ang 1-7-MAS axis in human diabetic nephropathy remains uncertain. Thus, we compared the renal ACE/ACE2-Ang 1-7-MAS expression of human diabetic nephropathy (DN) patients, healthy controls, and minimal change nephrotic syndrome (MCNS) patients.

\section{Methods}

In this observational case-control study, we investigated 17 patients with type 2 diabetes and overt diabetic nephropathy (DN), 17 healthy kidney transplant donors (healthy controls) who underwent renal biopsy during graft transplantation, and 11 patients with MCNS as disease controls, all of whom were admitted to Toho University Hospital between 2006-2010 and aged 20-65 years old. The inclusion criteria for overt diabetic nephropathy (DN) were based on the presence of type 2 diabetes, macroalbuminuria, and renal pathological findings compatible with diabetic nephropathy. All of our diabetic patients had type 2 diabetes, according to the criteria of The American Society of Diabetes Mellitus, and macroalbuminuria. Macroalbuminuria was defined as a urinary albumin-to-creatinine ratio of more than $300 \mathrm{mg} / \mathrm{gCr}$ in 2 specimens. DN was confirmed in all diabetic patients by pathological evaluation of renal biopsy specimens under a light microscope, immunofluorescent staining, and electron microscopy. The exclusion criteria for DN were the presence of renal disease other than DN and taking ACE inhibitors or angiotensin II type I (AT1) receptor blockers within 10 days prior to the renal biopsy. At the time of the renal biopsy, 14 patients were on antihypertensive agents, including 9 on calcium channel blockers; 2 on calcium channel blockers and alpha-blockers; 2 on calcium channel blockers

*Corresponding author: Sonoo Mizuiri, Division of Nephrology, Ichiyokai Harada Hospital 7-10, Kairoyama-cho, Saeki-ku, Hiroshima-City, 731-5134, Japan, Tel: +81 82-923-5161; Fax: +81 82 921-8035; E-mail: sm210@med.toho-u.ac.jp

Received January 23, 2012; Accepted March 15, 2012; Published March 17 2012

Citation: Mizuiri S, Nishizawa Y, Hamanoue M, Hemmi H, Arita M, et al. (2012) ACE2- Ang 1-7-MAS Axis in Human Diabetic Nephropathy. J Nephrol Therapeutic S2. doi:10.4172/2161-0959.S2-005

Copyright: ( 2012 Mizuiri S, et al. This is an open-access article distributed under the terms of the Creative Commons Attribution License, which permits unrestricted use, distribution, and reproduction in any medium, provided the original author and source are credited. 
and diuretics; and 1 on calcium channel blockers, alpha-blockers and diuretics, while 3 patients were not on any antihypertensive agents. None of the healthy controls or MCNS patients were being treated with antihypertensive agents. As for diabetes treatments, 8 patients were on oral antihyperglycemic agents ( 3 on glimepiride, 2 on glimepiride and voglibose, 3 on mitiglinide), 7 were receiving insulin injections, and 2 were being treated with diet therapy alone. Seven out of 8 patients on oral antihyperglycemic agents also received antihypertensive agents. Each subject's salt intake was standardized to $6 \mathrm{~g}$ daily during their hospitalization. Diagnosis of MCNS was made by using established criteria of clinical presentation of nephrotic syndrome at the time of renal biopsy, normal serum complement activity, no clinical or serologic evidence of collagen-vascular disease, relatively benign urine sediment, good response to steroid therapy and appropriate renal biopsy finding of minimal changes under light microscopy, no immunofluorescent immunoglobulin staining, and no marked changes except foot process effacement under electron microscopy. The exclusion criteria for MCNS is presence of taking medications at the time of renal biopsy. The medical records of all subjects from the time of the renal biopsy were reviewed to obtain data regarding age, sex, blood pressure, fasting blood sugar (FBS), hemoglobin Alc ( $\mathrm{HbA1c}$ ), proteinuria, serum albumin, and serum creatinine. The estimated glomerular filtration rate (eGFR) was calculated using the equations for estimated GFR together with a Japanese coefficient [10]. The study protocol was approved by the ethics committee of Toho University School of Medicine, and informed consent was obtained from all subjects.

\section{Immunofluorescent staining and confocal microscopy}

Double immunofluorescent staining of kidney sections for ACE and ACE2, Ang 1-7 and ACE2, or MAS and ACE2 was performed. The following primary antibodies were used for the immunofluorescent immunostaining: anti-human ACE goat antibody (AF929: R\&D Systems, Minneapolis, MN, USA), anti-human ACE2 mouse antibody (MAB933: R\&D Systems), anti-human Ang 1-7 rabbit antibody (H-002-24: Phoenix Pharmaceutical, Inc., Burlingame, California, USA), and anti-human MAS goat antibody (sc-54682: Santa Cruz Biotechnology, Heidelberg Germany). The specificities of the antibodies to human ACE and ACE2 were confirmed by Western blotting in our previous study [11]. The specificities of the antibodies to human Ang 1-7 and the human MAS were also confirmed by Western blotting at each supplier's laboratory. Paraffin-embedded kidney sections (4 $\mu \mathrm{m})$ were deparaffinized and rehydrated. After antigen retrieval, the sections were incubated overnight with the primary antibody diluted in $0.01 \mathrm{M}$ phosphate buffered saline (PBS) $(1: 100)$ at $4^{\circ} \mathrm{C}$. Then the sections were washed with Tris-buffered saline supplemented with Tween-20 three times and then incubated for $60 \mathrm{~min}$ with two of the respective secondary antibodies; Alexa Fluor (Invitrogen, Tokyo, Japan) 546 goat anti-mouse IgG (A-11030; highly cross-adsorbed), Alex Fluor 488 donkey anti-goat IgG (A-11055), or Alex Fluor 680 goat anti-rabbit IgG (A-21109; highly cross-adsorbed) diluted 1:200 in PBS and then sealed with Dapi-Fluoromount- $G^{\mathrm{TM}}$ (Southern Biotech, Birmingham, AL USA). Dapi-Fluoromount $-\mathrm{G}^{\mathrm{TM}}$ was used for sealant and nuclear staining by 4',6-diamidino-2-phenylindole (DAPI). The sections were visualized with a Zeiss LSM 510 confocal microscope (Carl Zeiss Microscopy, Jena, Germany). Negative controls for the immunofluorescence staining analysis were produced by substituting the primary antibodies with non-immune serum. In addition, the specificity of the staining obtained with Ang 1-7 was assessed by preabsorption of the antibody with $10 \mu \mathrm{mol} / \mathrm{L}$ Ang 1-7 (Bachem, Bubendorf, Switzerland).

\section{Immunohistochemical analysis of MAS expression in kidney specimens}

For MAS, immunohistochemistry was also performed using paraffin-embedded tissues by the indirect enzyme horseradish peroxidase-labeled antibody method with LSAB kit (Dako, Carpinteria, CA, USA). The primary antibody used was anti-human MAS1 goat antibody (sc-54682: Santa Cruz Biotechnology). Kidney sections (4$\mu \mathrm{m}$ thick) were deparaffinized and rehydrated. Antigen retrieval was performed with a pressure cooker at $98^{\circ} \mathrm{C}$ in target retrieval solution (Dako, Glostrup, Denmark). The sections were incubated overnight at $4^{\circ} \mathrm{C}$ with the anti-human MAS goat antibody (sc-54682: Santa Cruz Biotechnology), then washed with PBS and incubated with biotinylated universal (anti-rabbit, anti-mouse, anti-goat) IgG followed by incubation with peroxidase-labeled streptavidin. Peroxidase labeling was identified using a liquid diaminobenzidine substrate-chromogen and sections were counterstained with hematoxylin. Negative control tests were performed by omission of primary antibody and use of nonimmune sera.

\section{Semi quantitative analyses for MAS immunostaining}

The percentage and intensity of labeled surface area were evaluated by the method of Mezzano et al. [12], using a computer-based color image analysis system (Mac Scope v2.5, Mitani, Tokyo, Japan), which incorporates Code Warrior by Metrowerks (Austin, TX, USA). The intensity of staining was calculated from the ratio of suitable binary threshold imaging to the total region of interest (ROI), integrating staining intensity in specific areas. For each sample, a mean value was obtained for tubulointerstitial staining by analyzing all fields in a specimen excluding glomeruli and vessels, and the mean value for glomerular staining was obtained by analyzing all glomeruli in a specimen. The staining score was expressed as density/pixel.

\section{In situ hybridization analysis of MAS expression in human} kidney specimens

The oligonucleotide probes were derived from a 20 -mer sequence of human MAS-1 mRNA. The antisense and sense oligonucleotides for human MAS-1 corresponded to bases 651-670 of the MAS-1 gene [ACC. No. NM_02377]. Non-radioactive in situ hybridization was performed on paraffin-embedded sections from the same biopsy sample using reagents purchased from Pan Path (Amsterdam, Netherlands) as instructed by the manufacturer. Briefly, kidney sections $(4 \mu \mathrm{m}$ in thickness) were deparaffinized and rehydrated. After target retrieval, the slides were hybridized with digoxigenin-labeled oligonucleotide antisense probes $(100 \mu \mathrm{M})$ at $37^{\circ} \mathrm{C}$ for $16 \mathrm{~h}$. The probe was detected with nitro blue tetrazolium/5-bromo-4-chloro-3-indolyl phosphate (NBT/BCIP) and alkaline phosphatase-conjugated anti-digoxigenin antibodies. The sections were counterstained with nuclear Fast Red. The reaction specificity was confirmed by the disappearance of hybridization signals following incubation with the sense probes.

\section{PCR for human kidney MAS mRNA}

Total RNA was isolated from kidney biopsy specimens using the RNA easy Mini kit (Qiagen, Hilden, Germany). Samples of $1 \mu \mathrm{g}$ of total RNA were subjected to oligo-dT primed reverse transcription using Moloney murine leukemia virus reverse-transcriptase (Toyobo, Tokyo, Japan).The expression of MAS mRNA and $\beta$-actin mRNA were analyzed by reverse transcriptase-polymerase chain reaction (RTPCR). Specimens were amplified in a DNA Thermal Cycler (Eppendorf, Hamburg, Germany) with Taqman Universal PCR Mastermix and Taqman Gene Expression Assay kits for human MAS1, (Assay ID: 
Hs00267157_SI, Applied Biosystems Foster City, CA, USA) and for human $\beta$-actin (Assay ID: Hs00357333_g1, Applied Biosystems), respectively, according to the supplier's protocol. Thermal cycler conditions contained holds for two minutes at $50^{\circ} \mathrm{C}$ and ten minutes at $95^{\circ} \mathrm{C}$, followed 40 cycles of 15 seconds at $95^{\circ} \mathrm{C}$ and one minute at $60^{\circ} \mathrm{C}$. An aliquot of PCR product was then resolved by electrophoresis on $8 \%$ polyacrylamide gels and stained by ethidium bromide to visualize signal intensity. $\beta$-actin served as an internal RNA control to allow comparison of mRNA levels among different specimens but precise quantitative analysis by real time PCR was not performed in this study.

\section{Statistical analysis}

The data were not normally distributed, and non-parametric tests were performed in all analyses. Data are expressed as median values and $25-75 \%$ interquartile ranges (IQR). Differences among the three groups were assessed using the Kruskal-Wallis test and the post hoc Scheffe test. Gender based differences were assessed using the chisquare test. A P-value of less than 0.05 was considered to indicate a significant difference.

\section{Results}

The patients' clinical data are shown in Table 1. Compared with the healthy controls, the DN patients displayed significantly higher SBP, $\mathrm{DBP}, \mathrm{FBS}, \mathrm{HbA1c}$, proteinuria and serum creatinine levels, while their serum albumin and eGFR levels were significantly reduced. Compared with the MCNS patients, the DN patients displayed significantly higher SBP, DBP, FBS, HbA1c, serum creatinine, and serum albumin levels, while their proteinuria levels and eGFR were significantly reduced. There were no significant differences in age or the sex ratio among the three groups. Median and IQR value of serum creatinine was 1.1 [1.0$1.8] \mathrm{mg} / \mathrm{dl}, 0.8$ [0.6-1.0] $\mathrm{mg} / \mathrm{dl}$ and 0.8 [0.7-0.8] mg/dl in DN, MCNS and controls. Median value of proteinuria was 3.7 [1.9-4.7] g/day and 6.8 [6.0-10.2] g/day in DN and MCNS.

Localization of ACE, ACE2, Ang 1-7, and the MAS receptor using immunofluorescent staining and confocal microscopy

In our study, the double staining of ACE and ACE2, Alexa Fluor 488 and Alexa Fluor 546were conveniently green (ACE) and red

\begin{tabular}{|l|c|c|c|}
\hline & Healthy controls & MCNS & $\begin{array}{c}\text { Diabetic } \\
\text { nephropathy }\end{array}$ \\
\hline N [M/F] & $17[14 / 3]$ & $11[6 / 5]$ & $17[13 / 4]$ \\
\hline Age [years old] & $51(39-59)$ & $41(30-53)$ & $51(37-57)$ \\
\hline SBP [mmHg] & $118(112-124)$ & $122(113 / 128)$ & $150(132-156)^{* *} \dagger$ \\
\hline DBP [mmHg] & $74(70-80)$ & $72(70-78)$ & $78(76-82)^{*} \dagger$ \\
\hline FBS [mg/dl] & $97(92-100)$ & $95(92-100)$ & $163(115-197)^{\star *} \dagger \dagger$ \\
\hline HbA1c [\%] & $5.2(5.1-5.5)$ & $5.1(5.0-5.4)$ & $6.7(6.3-8.2)^{* *} \dagger$ \\
\hline Proteinuria [g/day] & $0(0-0)$ & $6.8(6.0-10.2)^{\star *}$ & $3.7(1.9-4.7)^{* *} \dagger$ \\
\hline Serum albumin [g/dl] & $4.5(4.2-4.7)$ & $1.7(1.3-2.1)^{* *}$ & $3.4(2.4-3.5)^{* *} \dagger \dagger$ \\
\hline Serum creatinine[mg/dl] & $0.8(0.7-0.8)$ & $0.8(0.6-1.0)$ & $1.1(1.0-1.8)^{*} \dagger$ \\
\hline eGFR [ml/min/1.73m²] & $87.2(71.3-95.7)$ & $71.8(49.2-100.2)$ & $49.8(23.7-62.1)^{*} \dagger$ \\
\hline
\end{tabular}

Data are shown as median values with interquartile ranges in parentheses. ${ }^{*} \mathrm{P}<0.05$ compared to the healthy controls, ${ }^{* *} \mathrm{P}<0.001$ compared to the healthy controls, $\uparrow \mathrm{P}<0.05$ compared to the MCNS patients, $\uparrow \uparrow \mathrm{P}<0.001$ compared to the MCNS patients

SBP: systolic blood pressure, DBP: diastolic blood pressure, FBS: fasting blood sugar

MCNS: Minimal change nephrotic syndrome

Table 1: Clinical data.



Figure 1: Immunofluorescent staining of angiotensin-converting enzyme (ACE; green), ACE2 (red), and a nuclear marker (DAPI; blue) in kidney specimens $(A, B, C)$. Merged images of ACE and ACE2 (yellow) staining showing the colocalization of ACE and ACE2 in the proximal tubules of a healthy control (A) and an MCNS (C) patient. The merged images for the diabetic nephropathy patient do not include the yellow signals reflecting weak ACE2 staining (B).
A: Healthy controls

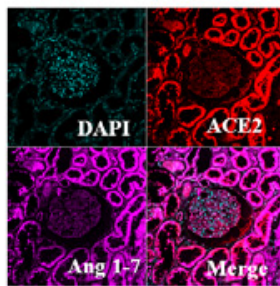

B: Diabetic nephropathy

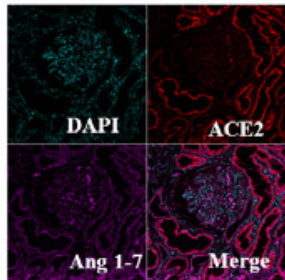

C: MCNS

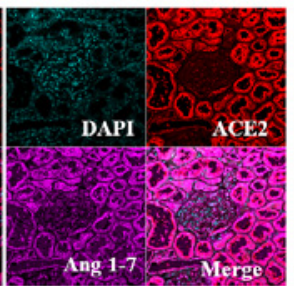

Figure 2: Immunofluorescent staining of angiotensin 1-7 (Ang 1-7; purple), ACE2 (red), and a nuclear marker (DAPI; blue) in kidney specimens (A, B, C). Merged images of Ang 1-7 and ACE2 (pink) staining showing the co-localization of ACE and ACE2 in the proximal tubules of a healthy control and an MCNS patient $(A, C)$. The merged image of the proximal tubules of the diabetic nephropathy patient $(B)$ is a completely different color from that of the healthy control $(A)$.

(ACE2), respectively, and overlapping domains of expression were yellow (Figure 1). In the healthy subjects, ACE and ACE2 displayed co-localization (yellow) in the proximal tubules (Figure 1A). ACE and ACE2 also displayed weak staining in the glomeruli (Figure 1A). In contrast with healthy subjects, ACE2 staining was extremely decreased in both tubules and glomeruli in DN patients (Figure 1B). The merge of both ACE and ACE2 images showed green color but not yellow in the proximal tubules (Figure 1B, lower right). The MCNS patients displayed similar ACE and ACE2 staining patterns to the healthy controls (Figure 1C).

In the double staining of Ang 1-7 and ACE2, Alexa Fluor 680 and Alexa Fluor 546were conveniently purple (Ang 1-7) and red (ACE2), respectively, and overlapping domains of expression were pink (Figure 2). Ang 1-7 was detected in the proximal tubules without marked staining in the glomeruli in all three groups (Figure 2A,B,C). As shown in merged image's pink color, Ang 1-7 and ACE2 co-localized in the proximal tubules in the healthy controls and MCNS patients (Figure $2 \mathrm{~A}, \mathrm{C}$ ), but proximal tubules in merged images did not show pink color in tubules of DN patients (Figure 2B). Tubular Ang 1-7 expression was downregulated in the diabetic kidneys compared with those of the healthy controls and MCNS patients (Figure 2B).

In the double staining of MAS and ACE2, Alexa Fluor 488 and Alexa Fluor 546 was conveniently green (MAS) and red (ACE2), respectively, and overlapping domains of expression were yellow (Figure 3). MAS were detected in the proximal tubules and displayed weak staining in the glomeruli in all three groups (Figure $3 \mathrm{~A}, \mathrm{~B}$, and C). As shown in merged image's yellow color, MAS and ACE2 also co localized in the proximal tubules in the healthy controls and MCNS patients. (Figure 
3A and C). The merge of MAS and ACE2 images did not show yellow color in the proximal tubules of DN patients (Figure 3B, lower right). Compared with the healthy controls, the MCNS patients displayed a similar tubular MAS expression level (Figure 3C), whereas the DN patients demonstrated downregulated tubular and glomerular MAS expression (Figure 3B).

\section{Immunohistochemical analysis of MAS receptor expression}

Images of immunohistochemical staining of the MAS receptor in representative kidney sections from a healthy control, a patient with diabetic nephropathy, and an MCNS patient are shown in Figure 4. Compared with the kidney specimen from the healthy control, the MCNS patient displayed a similar level of MAS receptor staining, whereas the DN patient's proximal tubules and glomeruli only displayed weak staining (Figure 4).

Table 2 details the results of our semiquantitative analysis of immunohistochemical renal MAS protein expression. Tubular MAS expression (density/pixel) was significantly $(\mathrm{P}<0.001)$ downregulated in the DN patients [7.33 (6.26-9.68)] compared with the healthy controls [24.51 (18.06-34.56)] and MCNS patients [23.75 (20.11-25.52)]. Glomerular MAS expression (density/pixel) was also downregulated in the DN patients 0.84 (0.13-1.69) compared with healthy controls [3.26 (2.36-6.65), $\mathrm{p}<0.05]$ and MCNS patients [4.02 (2.47-6.86)], although the difference between DN patients and MCNS patients was not significant. No significant difference in tubular or glomerular MAS staining was detected between the MCNS patients and healthy controls.

\section{Human kidney MAS mRNA expression}

MAS mRNA was detected in both the proximal tubules and glomeruli in all subjects by in situ hybridization, but tubular MAS mRNA expression was markedly decreased in the kidneys of the DN patients compared with the kidneys of the healthy controls and

A: Healthy control

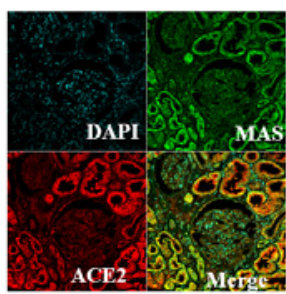

B: Diabetic nephropathy

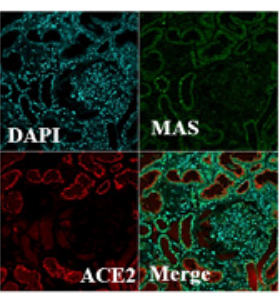

C: MCNS

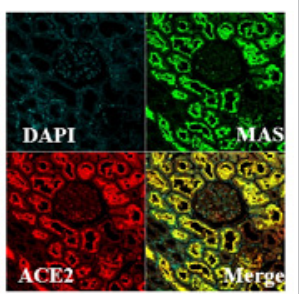

Figure 3: Immunofluorescent staining of the MAS receptor (MAS; green), ACE2 (red), and a nuclear marker (DAPI; blue) in kidney specimens (A, B, C). MAS staining was observed in both the tubules and glomeruli $(A, B, C)$. Merged images of MAS and ACE2 (yellow) staining showing the co-localization of ACE and ACE2 in the proximal tubules of a healthy control and an MCNS patient $(A, C)$. The merged imaged of the proximal tubules of the diabetic nephropathy patient do not show yellow signals (B) reflecting weak ACE2 and MAS staining.

A: Healthy control

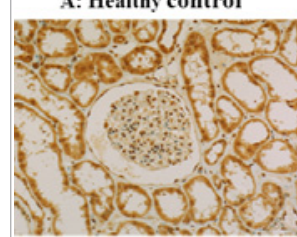

Figure 4: Immunostaining of the MAS receptor in kidney specimens from a healthy control (A), a patient with diabetic nephropathy (B), and a patient with MCNS (C). The MAS staining was weak in the specimen from the DN patient (B)

\begin{tabular}{|l|c|c|c|}
\hline & Healthy controls & MCNS & $\begin{array}{l}\text { Diabetic } \\
\text { nephropathy }\end{array}$ \\
\hline $\mathrm{N}$ & 17 & 11 & 17 \\
\hline $\begin{array}{l}\text { Protein staining } \\
\text { [density/pixel] }\end{array}$ & & & \\
\hline $\begin{array}{l}\text { Tubulointerstitial } \\
\text { MAS receptor }\end{array}$ & $24.51(18.06-34.56)$ & $23.75(20.11-25.52)$ & $7.33(6.26-9.68)^{\star *}+$ \\
\hline $\begin{array}{c}\text { Glomerular } \\
\text { MAS receptor }\end{array}$ & $3.26(2.36-6.65)$ & $4.02(2.47-6.86)$ & $0.84(0.13-1.69)^{\star}$ \\
\hline
\end{tabular}

Data are shown as median values with interquartile ranges in parentheses.

${ }^{*} \mathrm{P}<0.05$ compared to the healthy controls, ${ }^{* *} \mathrm{P}<0.001$ compared to the healthy controls, $\dagger P<0.001$ compared to the MCNS patients

MCNS: Minimal change nephrotic syndrome

Table 2: Semiquantitative immunohistochemical analysis of MAS receptor expression.

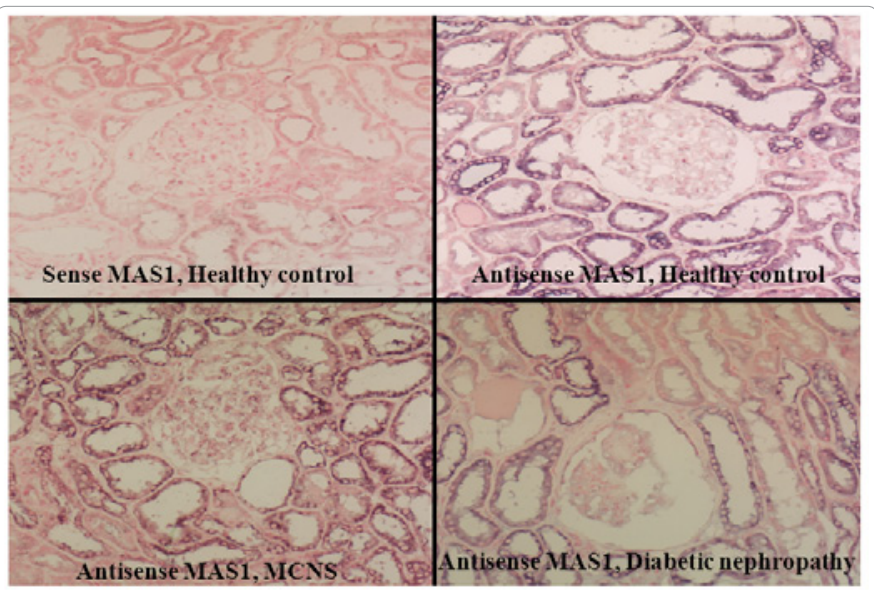

Figure 5: In situ hybridization of the MAS receptor in human kidney specimens. MAS receptor mRNA expression was downregulated in the kidneys from the diabetic nephropathy patients (right lower panel) compared with those from the other subjects. Negative control test on a kidney specimen with the sense probes (left upper panel).

MCNS patients (Figure 5). Negative control tests using a sense probe confirmed the specificity of the assay (Figure 5, upper left).

MAS mRNA was also detected by RT-PCR, although MAS mRNA expression was suppressed in the kidney extract from the DN patients compared with the kidney extracts from the healthy controls and MCNS patients (Figure 6).

\section{Discussion}

Diabetes is known to activate the RAS in the kidney [13,14]. Previously, we and others have suggested that the combination of increased ACE expression and decreased ACE2 expression augment Ang II production, leading to accelerated glomerular injury in human type 2 diabetic nephropathy $[4,11,15,16]$. However, no reports on renal ACE and ACE2 in the same specimen by double staining have been reported in human type 2 diabetic nephropathy. Furthermore none of them studied angiotensin 1-7, MAS in human diabetic nephropathy with ACE2 in the same kidney specimens. Our study was performed to clarify the co localization of ACE2, Ang 1-7 and MAS in human kidney in the same specimen by double immunofluorescent staining and confocal microscopy to identify the significance of ACE2-Ang 1-7-MAS Axis in human type 2 diabetic nephropathy. In contrast with healthy subjects and MCNS patients, proximal tubules in merged images using confocal microscopy from DN patients did not show yellow color indicating co-localization of ACE and ACE2, MAS and ACE2, nor pink color indicating co-localization of Ang 1-7 and ACE2. 


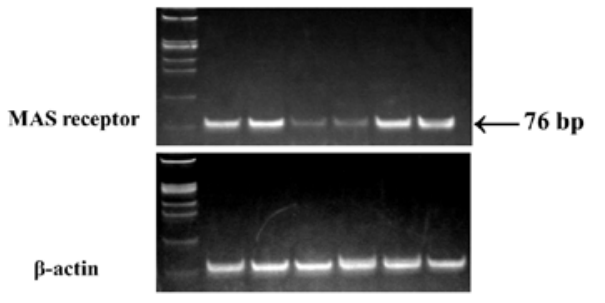

Figure 6: MAS receptor mRNA expression according to RT-PCR in human renal biopsy samples. Lane 1: size marker; Lanes 2 and 3: healthy controls; Lanes 4 and 5: patients with diabetic nephropathy; Lanes 6 and 7: patients with minimal change nephrotic syndrome. The 76-bp PCR product is the MAS receptor. Decreased mRNA expression was observed in the samples from the DN patients.

However, this may be derived from extremely decreased ACE2 staining but not from different localization of these proteins and ACE2 in DN patients.

ACE2 plays a significant role in modulating the RAS by regulating a number of angiotensin molecules including Ang I, Ang II, Ang 1-7, and Ang 1-9. In general, it is thought that activation of the ACE2-Ang 1-7-MAS axis counteracts the actions of the ACE-Ang II-AT1 axis by inhibiting MAP kinase-or cyclooxygenase-2 (COX-2)-dependent pathways, or stimulating the nitric oxide (NO)-cGMP-dependent pathway $[5,6,17]$.

However, Shalamanova et al. [18] reported that they could not to detect MAS mRNA expression in the human kidney, primary human renal proximal tubular cells, or human proximal tubular epithelial (HK-2) cells by RT-PCR, although they did detect it in rat primary proximal tubular cells. Thus, they suggested that the expression of MAS in the kidney might be species-specific [18]. In cultured human proximal tubular cells and human mesangial cells, MAS protein expression has been detected by immunoblot experiments $[4,19]$, and we have also detected MAS protein by immunofluorescent staining and immunohistochemistry and MAS mRNA by in situ hybridization and RT-PCR in human kidney biopsy specimens. The discrepancy between Shalamanova's findings and ours might have been caused by the differences in the primers and PCR conditions used, and we are confident that the MAS is present in human kidneys.

In all subjects in our study, immunofluorescent and immunohistochemical staining of MAS revealed that it is predominantly expressed in the proximal tubules and weakly expressed in the glomeruli. With regard to the presence of MAS in the glomeruli, some immunofluorescent staining study did not detect MAS protein in the glomeruli [20], while others detected immune reactive signals in the glomeruli of the rat kidney [21] or human mesangial cells [19]. Our study suggests that MAS is expressed in the glomeruli in addition to proximal tubules.

Our study showed using confocal microscopy and double immunofluorescent staining that both Ang 1-7 and the MAS colocalized with ACE2 in the proximal tubules of healthy human kidneys. Moreover, we detected the glomerular localization of ACE, ACE2, and MAS although these proteins were not always colocalized, and glomerular staining of Ang 1-7 was not evident in our immunofluorescent study. The results on Ang 1-7 should be interpreted with caution, since, Ang 1-7 is a bioactive transmitter. The concentration of bioactive transmitter (Ang 1-7) may be difficult to demonstrate by immunofluorescent staining because the half-life is usually calculated by seconds even if it is localized in glomeruli. Our immunofluorescent study detected decreased ACE2, Ang 1-7, and MAS staining in the proximal tubules of DN patients compared with those of healthy subjects and MCNS patients. Semiquantitative analysis of MAS immunostaining also detected significantly decreased MAS expression in the tubules and glomeruli of the DN patients compared with the healthy controls. Compared to the MCNS patients, the DN patients displayed significantly decreased MAS immunostaining in their tubules. It was also decreased in their glomeruli although the difference was not statistically significant. The kidneys of the healthy controls and MCNS patients displayed similar ACE, ACE2, and MAS staining patterns in our double immunofluorescent staining experiment. In addition, our semiquantitative analysis of MAS immunostaining did not detect a significant difference between the healthy controls and MCNS patients. Our results are consistent with those of previously reported animal studies. For example, a Western blotting study showed that STZ-induced diabetic mice displayed reduced levels of Ang 1-7, the MAS receptor, and ACE2 compared with normal mice [22]. Using RT-PCR, Shao et al. [23] also reported that MAS receptor mRNA expression was significantly decreased in diabetic rats compared to the controls.

The ACE-Ang II-AT1 axis acts to raise blood pressure, induce renal vasoconstriction, decrease renal blood flow $(\mathrm{RBF})$ and the glomerular filtration rate (GFR), stimulate proximal tubule sodium transport, and increase urine concentration [5,17]. Conversely, the ACE2-Ang 1-7MAS axis acts to reduce blood pressure, dilate intrarenal blood vessels, increase RBF and GFR, inhibit proximal tubule transport, and induce diuresis $[5,17,24]$. The ACE-Ang II-AT1 axis is widely implicated in the pathogenesis and progression of diabetic nephropathy [4,17]. ACE2 overexpression to increase Ang 1-7 production or stimulation of the MAS by Ang 1-7 in the kidneys might act to counter the pathological role of the ACE-Ang II-AT1 axis [25,26]. However, the role of Ang 1-7 in the progression of diabetic nephropathy is not fully understood and might be cell specific [4]. It is reported that Ang 1-7 protects against endothelial function deterioration and Ang II-stimulated proximal tubular injury [6], although Ang 1-7 also activates growth stimulatory pathways associated with the production of TGF- $\beta 1$ and extracellular matrix proteins in cultured human mesangial cells [19]. Benter et al. [27] suggested that Ang 1-7 plays a renoprotective role against the development of diabetic nephropathy. Moon et al. [28] also reported that Ang 1-7 had an attenuating effect on angiotensin IImediated NAD $(\mathrm{P}) \mathrm{H}$ oxidase activation in type 2 diabetic nephropathy in mice. While, Shao et al. [23] reported that chronic Ang 1-7 injection accelerates STZ-induced diabetic renal injury. Little is known about the roles of Ang 1-7 and MAS in human diabetic nephropathy, but we consider that the downregulation of ACE2 and the MAS might limit the biological effects of Ang 1-7.

The DN patients' kidney biopsy samples displayed decreased ACE2, Ang 1-7, and MAS expression levels compared with those of the controls but not those of the MCNS patients. Both genetic ablation of ACE2 [29] and pharmacological inhibition [16] of ACE2 in diabetic mice increase albuminuria. However, the fact that the two proteinuric renal diseases examined in this study did not display similar renal ACE2, Ang 1-7, and MAS expression levels might have been due to differences in the pathogeneses and durations of these diseases. Considered together with previous reports on ACE2-Ang 1-7-MAS in proximal tubules $[6,27,28]$, our fining suggest that this decrease is not solely related to proteinuria. 
The present study has certain limitations. The number of human renal biopsy specimens that displayed structural distortion of cortical tissue was relatively small. Furthermore, 14 of the $17 \mathrm{DN}$ patients were being treated with antihypertensive agents including $\mathrm{Ca}$ channel blockers, alpha-blockers, and/or diuretics, which might have influenced the intrarenal RAS.

\section{Conclusion}

ACE/ACE2-Ang 1-7-MAS co-localize in human proximal tubules. The tubular ACE2-Ang 1-7-MAS axis is downregulated in human diabetic nephropathy patients compared with healthy controls and MCNS patients.

\section{Acknowledgment}

The authors thank Mr. Naoji Masuya of Toho University School of Medicine for providing expert statistical advice. This study was presented at the Renal Week Conference (American Society of Nephrology) in November 2010, Denver, CO, USA.

\section{References}

1. Tipnis SR, Hooper NM, Hyde R, Karran E, Christie G, et al. (2000) A human homolog of angiotensin-converting enzyme: Cloning and functional expression as a captopril-insensitive carboxypeptidase. J Biol Chem 275: 33238-33243.

2. Donoghue M, Hsieh F, Baronas E, Godbout K, Gosselin M, et al. (2000) A novel angiotensin-converting enzyme-related carboxypeptidase (ACE2) converts angiotensin I to angiotensin 1-9. Circ Res 87: 1-9.

3. Santos RA, Ferreira AJ, Simões E Silva AC (2008) Recent advances in the angiotensin-converting enzyme 2-angiotensin(1-7)-Mas axis. Exp Physiol 93: 519-527

4. Dilauro M, Burns KD (2009) Angiotensin-(1-7) and its effects in the kidney. ScientificWorldJournal 9: 522-535.

5. Ferrario CM, Varagic J (2010) The ANG 1-7/ACE2/mas axis in the regulation of nephron function. Am J Physiol Renal Physiol 298: 1297-1305.

6. Su Z, Zimpelmann J, Burns KD (2006) Angiotensin-(1-7) inhibits angiotensin II-stimulated phosphorylation of MAP kinases in proximal tubular cells. Kidney Int 69: 2212-2218.

7. Joyner J, Neves LA, Granger JP, Alexander BT, Merrill DC, et al. (2007) Temporal-spatial expression of ANG 1-7 and angiotensin-converting enzyme 2 in the kidney of normal and hypertensive pregnant rats. Am J Physiol Regul Integr Comp Physiol 293: 169-177.

8. Dilauro M, Zimpelmann J, Robertson SJ, Genest D, Burns KD (2010) Effect of ACE2 and angiotensin-(1-7) in a mouse model of early chronic kidney disease. Am J Physiol Renal Physiol 298: 1523-1532.

9. Batlle D, Wysocki J, Soler MJ, Ranganath K (2012) Angiotensin-converting enzyme 2: enhancing the degradation of angiotensin II as a potential therapy for diabetic nephropathy. Kidney Int 81: 520-528

10. Matsuo S, Imai E, Horio M, Yasuda Y, Tomita K, et al. (2009) Revised equations for estimated GFR from serum creatinine in Japan. Am J Kidney Dis 53: 982 992.

11. Mizuiri S, Hemmi H, Arita M, Ohashi Y, Tanaka Y, et al. (2008) Expression of $A C E$ and $A C E 2$ in individuals with diabetic kidney disease and healthy controls. Am J Kidney Dis 51: 613-623.

12. Mezzano S, Droguett A, Burgos ME, Ardiles LG, Flores CA, et al. (2003) Reninangiotensin system activation and interstitial inflammation in human diabetic nephropathy. Kidney Int Suppl 86: 64-70.

13. Lai KN, Leung JCK, Tang SCW (2011) The Renin-Angiotensin System. La KN, Tang SCW (eds): Diabetes and the Kidney. Contrib Nephrol. 170: 135-144.

14. Giacchetti G, Sechi LA, Rilli S, Carey RM (2005) The renin-angiotensinaldosterone system, glucose metabolism and diabetes. Trends Endocrinol Metab 16:120-126

This article was originally published in a special issue, Diabetic

Nephropathy handled by Editor(s). Dr. Jian-Kang Chen, Vanderbilt

University, USA
15. Reich HN, Oudit GY, Penninger JM, Scholey JW, Herzenberg AM, et al. (2008) Decreased glomerular and tubular expression of ACE2 in patients with type 2 diabetes and kidney disease. Kidney Int 74: 1610-1616.

16. Ye M, Wysocki J, William J, Soler MJ, Cokic I, et al. (2006) Glomerula localization and expression of Angiotensin-converting enzyme 2 and Angiotensin-converting enzyme: implications for albuminuria in diabetes. Am Soc Nephrol 17: 3067-3075.

17. Zhuo JL, Li XC (2011) New insights and perspectives on intrarenal reninangiotensin system: focus on intracrine/intracellular angiotensin II. Peptides 32: $1551-1565$

18. Shalamanova L, Wilkinson MC, McArdle F, Jackson MJ, Rustom R (2010) Characterisation of the expression of the Renin-Angiotensin system in primary and immortalised human renal proximal tubular cells. Nephron Exp Nephro 116: 53-61.

19. Zimpelmann J, Burns KD (2009) Angiotensin-(1-7) activates growth-stimulatory pathways in human mesangial cells. Am J Physiol 296: 337-346.

20. Chappell MC, Modrall JG, Diz DI, Ferrario CM (2004) Novel aspects of the renal renin-angiotensin system: angiotensin-(1-7), ACE2 and blood pressure regulation. Contrib Nephrol 143: 77-89.

21. Da Silveira KD, Pompermayer Bosco KS, Diniz LR, Carmona AK, Cassali GD, et al. (2010) ACE2-angiotensin-(1-7)-Mas axis in renal ischemia/reperfusion injury in rats. Clin Sci (Lond) 119: 385-394.

22. Lakshmanan AP, Watanabe K, Thandavarayan R, Sari F, Harima M, et al. (2011) Telmisartan attenuate oxidative stress and renal fibrosis in streptozotocin induced diabetic mice with the alternation of angiotensin-(1-7) mas receptor expression associated with its PPAR-y agonist action. Free Radic Res 45: 575-584.

23. Shao Y, He M, Zhou L, Yao T, Huang Y, et al. (2008) Chronic angiotensin (1-7) injection accelerates STZ-induced diabetic renal injury. Acta Pharmacol Sin 29: 829-837.

24. Chappell MC (2007) Emerging evidence for a functional angiotensin-converting enzyme 2-angiotensin-(1-7)-MAS receptor axis: more than regulation of blood pressure? Hypertension 50: 596-599.

25. Liu CX, Hu Q, Wang Y, Zhang W, Ma ZY, et al. (2011) Angiotensin-converting enzyme (ACE) 2 overexpression ameliorates glomerular injury in a rat mode of diabetic nephropathy: a comparison with ACE inhibition. Mol Med 17: 59-69.

26. Oudit GY, Liu GC, Zhong J, Basu R, Chow FL, et al. (2010) Human recombinan ACE2 reduces the progression of diabetic nephropathy. Diabetes 59: 529-538.

27. Benter IF, Yousif MH, Dhaunsi GS, Kaur J, Chappell MC, et al. (2008) Angiotensin-(1-7) prevents activation of NADPH oxidase and renal vascular dysfunction in diabetic hypertensive rats. Am J Nephrol 28: 25-33.

28. Moon JY, Tanimoto M, Gohda T, Hagiwara S, Yamazaki T, et al. (2011) Attenuating effect of angiotensin-(1-7) on angiotensin II-mediated NAD(P)H oxidase activation in type 2 diabetic nephropathy of $\mathrm{KK}-\mathrm{A}(\mathrm{y}) / \mathrm{Ta}$ mice. Am J Physiol Renal Physiol 300: 1271-1282.

29. Wong DW, Oudit GY, Reich H, Kassiri Z, Zhou J, et al. (2007) Loss of angiotensin-converting enzyme-2 (Ace2) accelerates diabetic kidney injury. Am J Pathol 171: 438-451. 